\title{
Structural dynamic modifications via models
}

\author{
T K KUNDRA
}

\author{
Mechanical Engineering Department, Indian Institute of Technology, New \\ Delhi, India \\ e-mail: tkkundra@mech.iitd.ernet.in
}

\begin{abstract}
Structural dynamic modification techniques attempt to reduce dynamic design time and can be implemented beginning with spatial models of structures, dynamic test data or updated models. The models assumed in this discussion are mathematical models, namely mass, stiffness, and damping matrices of the equations of motion of a structure. These models are identified/ extracted from dynamic test data viz. frequency response functions (FRFs). Alternatively these models could have been obtained by adjusting or updating the finite element model of the structure in the light of the test data. The methods of structural modification for getting desired dynamic characteristics by using modifiers namely mass, beams and tuned absorbers are discussed.
\end{abstract}

Keywords. Structural dynamic modification; dynamic design; spatial model; modal model; frequency response function.

\section{Introduction}

Structural dynamic modification (SDM) techniques can be defined as the methods by which dynamic behaviour of a structure is improved by predicting the modified behaviour brought about by adding modifications like those of lumped masses, rigid links, dampers, beams etc. or by variations in the configuration parameters of the structure itself. Such methods, especially those with their roots in finite element models, have often been described as REANALYSIS, see for example the surveys by Baldwin \& Hutton (1985), Snyder (1985) and the text on SDM by Brandon (1990). Most of the techniques imply a dynamic test at some stage of SDM and currently prefer implementation on a personal computer.

The need for SDM arises because of the demands on higher performance capabilities of complex mechanical and structural systems, like machine tools, automobiles, rail vehicles, aerospace systems and high speed rotating systems, which require sound dynamic design, i.e. desired dynamic characteristics like vibration levels/response, resonances/eigenvalues, dynamic stability and mode shapes. Dynamic design has been generally improved by timeconsuming and expensive intuitive cut-and-try prototype testing including those cases where FEM has limitations. Accordingly it is desirable to devise methods by which prototype modification cycles/design development time can be reduced. This is achieved in SDM by first predicting accurately and quickly at computer level the effect of possible 
modifications like addition of masses, beams and dampers etc. or of configuration changes on the dynamic characteristics of the structure, and then effecting modifications. The reanalysis research is directed towards techniques leading to quicker and, for that reason, more efficient prediction of the desired components of the modified dynamic behaviour. The techniques to predict dynamic behaviour of structures built from substructures by the substructuring or building block approach, which is an area by itself, however, have some similar principles which are not included in this discussion. The reader is referred to Craig (1985) for a review.

Another important objective of SDM is the prediction of the magnitudes and locations of modifications or structural variations for shifting the resonances or improving the other parameters of dynamic performance. This objective is obviously the inverse of the analytical objective mentioned above and could be thus viewed as something more like design synthesis. The methods of sensitivity analysis of the structure's eigenvalues and eigenvectors with respect to modification parameters help achieve this objective. These methods naturally look further for optimal modifications and accordingly embrace several of structural dynamic optimization techniques. A review of structural optimization in vibratory environments is given by Rao (1989).

\section{SDM techniques}

SDM methods may be broadly divided into two groups. Those which employ a model of the structure and those that use dynamic test data directly.

The model used by the first type of SDM techniques may be physical or spatial (mass, stiffness and damping parameters) or a modal model (natural frequencies, mode shapes and damping coefficients). The model's parameters are extracted from dynamic test data of a structure by using system identification and model testing methods as described by Berman (1975), Natke (1982) and Ewins (1984). The model could also be an updated model which is invariably a finite element model adjusted and corrected in the light of experimental data. Construction of the latter type of model, often with spatial properties has been reviewed by Imregun \& Visser (1991).

The second type of SDM techniques use the experimental data directly rather than the above mentioned initial system or modal identification or model updating. The dynamic test used could be for receptance, mobility or inertance and is called frequency response function (FRF).

Both the above techniques assume the existence of a structure which requires dynamic performance improvement or, more appropriately, troubleshooting. Alternatively, a prototype is available for dynamic testing of the structure to achieve sound dynamic design. The major part of this paper is devoted to SDM via models.

\section{SDM via models}

The dynamic behaviour of a structure which is assumed to be linear and approximately discretized for $n$ degrees of freedom (DOF) can be described by the equations of motion,

$$
[M]\{\ddot{x}\}+[C]\{\dot{x}\}+[K]\{x\}=\{f\},
$$


where $[M],[K]$ and $[C]$ are mass, stiffness and damping matrices of the structure, and with hysteretic damping given by

$$
[M]\{\ddot{x}\}+([K]+i[H])\{x\}=\{f\},
$$

where $[H]$ is the hysteretic damping matrix.

The eigenvalues for the structure are predicted by solving

$$
([K]-\lambda[M])\{x\}=0 .
$$

The structural modifications cause changes in the parameter matrices of the spatial model of the structure and the above equations get modified as (Kundra 1986).

$$
([M]+[\mathrm{d} M])\{\ddot{x}\}+([C]+[\mathrm{d} C])\{\dot{x}\}+([K]+[\mathrm{d} K])\{x\}=\{f\}
$$

where $[\mathrm{d} M],[\mathrm{d} K]$ and $[\mathrm{d} C]$ are the changes in the parametric matrices due to modification.

$$
\left([K]+[\mathrm{d} K]-\lambda_{m}([M]+[\mathrm{d} M])\right)\{x\}=0 .
$$

In modal coordinates $(\{x\}=[\phi]\{p\})$ the equations can be written as (Luk \& Mitchell 1984)

$$
\left.\left[M_{m}\right]\{\ddot{p}\}+\left[C_{m}\right]\right)\{\dot{p}\}+\left[K_{m}\right]\{p\}=[\phi]^{T}\{f\},
$$

where

$$
\begin{aligned}
{[\phi] } & =\text { mass normalized eigenvector matrix, } \\
{\left[M_{m}\right] } & =[\phi]^{T}[M][\phi]+[\phi]^{T}[\mathrm{~d} M][\phi], \\
{\left[K_{m}\right] } & =[\phi]^{T}[K][\phi]+[\phi]^{T}[\mathrm{~d} K][\phi], \\
{\left[C_{m}\right] } & =[\phi]^{T}[C][\phi]+[\phi]^{T}[\mathrm{~d} C][\phi] .
\end{aligned}
$$

The first terms in the set of (7) are equal to the diagonalized modal mass stiffness and damping matrices and can be obtained by experimental modal analysis (Kundra 1986). Further, in (7), $[\phi]$ can be an $n \times m$ matrix permitting experimental data about $m$ modes only where $m<n$, as explained by Ewins (1984). Accordingly, $\left[M_{m}\right]$ etc. would be of $m \times m$ size.

The equations (4-6) used for predicting dynamic behaviour of the modified structure using spatial or modal models imply that matrix changes $[\mathrm{d} M]$, $[\mathrm{d} K]$ and $[\mathrm{d} C]$ or $[\mathrm{d} H]$ are determined, in the same coordinates as those of the original matrices and configuration variations of the structure. Accordingly, it is one of the important issues addressed in model-based SDM techniques.

\subsection{Mass modifications}

The determination of $[\mathrm{d} M]$ in case of modification of a structure with a lumped mass $m$ is straightforward and can be obtained by inserting the modifying mass $m$ at diagonal locations of the matrix with zero elements. The locations are determined by the coordinates of motion influenced by modifying mass $m$.

For instance in the F letter type structure shown in figure 1, which is simulated as an eight-degree of freedom (DOF) system (figure 2), if mass $m$ (say a motor on a machine tool) 


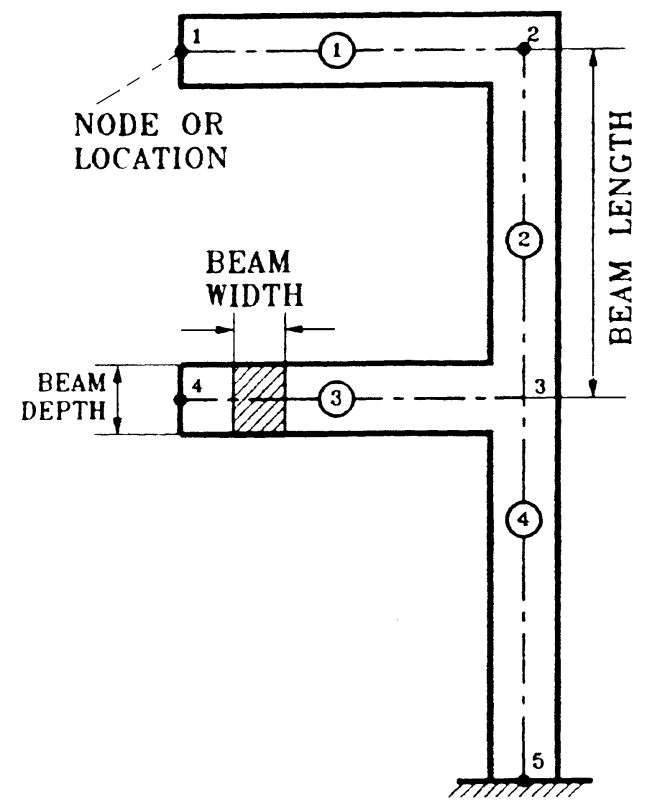

Figure 1. F-type structure.

is shifted from location 2 to location 1 , the modification matrix would be

$$
[\mathrm{d} M]=\left[\begin{array}{cccccccc}
+m & 0 & 0 & 0 & 0 & 0 & 0 & 0 \\
0 & +m & 0 & 0 & 0 & 0 & 0 & 0 \\
0 & 0 & -m & 0 & 0 & 0 & 0 & 0 \\
0 & 0 & 0 & -m & 0 & 0 & 0 & 0 \\
0 & 0 & 0 & 0 & 0 & 0 & 0 & 0 \\
0 & 0 & 0 & 0 & 0 & 0 & 0 & 0 \\
0 & 0 & 0 & 0 & 0 & 0 & 0 & 0 \\
0 & 0 & 0 & 0 & 0 & 0 & 0 & 0
\end{array}\right] .
$$

It can be observed that removal of mass at location 2 affects coordinates 3 and 4 and implies negative entries of mass while addition of mass at location 1 brings in positive masses at the affected coordinates 1 and 2.

\subsection{Modifying material of structure}

Hardware changes affecting the material of the entire structure can be assumed in several cases to affect the damping matrix only. Whenever the mass matrix is also affected because of significant density differences of the materials, the mass modification matrix may be obtained as explained earlier by lumping the modifying masses. The modified structural damping matrix $[H]_{M}$ is obtained as

$$
[H]_{M}=[H]+[\mathrm{d} H],
$$

where

$$
[\mathrm{d} H]=g_{c}[K] .
$$


If $g_{m}$ is the desired overall structural damping coefficient of the structure with new proposed material then,

$$
g_{c}=g_{m}-g_{a v},
$$

where $g_{a v}$ is obtained as the average of the identified damping coefficients of several modes of the system. In general,

$$
[H]=g_{i}[K]
$$

if structural damping coefficient $g_{i}$ is same for all the modes.

\subsection{Stiffness and beam modifications}

For pure stiffness modifiers $[\mathrm{d} K]$ between nodes can be identified on lines of mass modifications and is simple in many cases. Providing a pure stiffness in real design practice of course is not easy. Accordingly, difficulties arise in evaluating $[\mathrm{d} K]$ when beam-like modifications, as required in design practice, have to be made on structures with the intention of stiffening them. Spatial model-based modification of a structure with beams has been studied and it is observed that it influences all the parameter matrices in a complex manner. Some numerical approaches to get approximate values of the modified matrices have been proposed (Kundra 1986). Luk \& Mitchell (1984) proposed modifications in dual spaces for pure masses and stiffnesses. The difficulties encountered in a typical case and an approach to overcome the same are presented below.

A hardware modification like that of alternating one of the structural elements of a system could be too complex to be interpreted in terms of the modified matrices. For example, attachment of a rib-like stiffener between locations 1 and 2 of the F-type structure shown in figure 1 , influences mass and stiffness, as well as the damping matrices. Though the modified mass matrix can be obtained by lumping the mass of the stiffener at the nodes, it is not easy to obtain the modified stiffness and damping matrices. The parameters of the modified/modifying structural element affect the stiffness matrix in a highly complex manner. It is particularly so due to the difficulties of measuring accurately the rotational responses and thereby including those in the equations of motion. For instance, the reduced stiffness matrix for one plane motion simulation of the F structure taking due care of the rotational degrees of freedom can be analytically obtained as,

$$
[K]=\left[K_{A A}\right]=\left[K_{A B}\right]\left[K_{B B}\right]^{-1}\left[K_{B A}\right],
$$

where

$$
\left[\begin{array}{ll}
{\left[K_{A A}\right]} & {\left[K_{A B}\right]} \\
{\left[K_{B A}\right]} & {\left[K_{B B}\right]}
\end{array}\right]
$$

is the partitioned matrix in the equation relating the excitation forces and the displacement vectors in the simulated F-type structure (figure 2). The analytical expressions for the elements of the reduced stiffness matrix $[K]$ show that the matrix elements are influenced by the parameters of several beam elements of the structure in a complex manner. This is also true of other affected elements of the matrix $[K]$. The study shows that as many as half of the matrix elements of $[K]$ get affected when a particular beam element of the structure is modified. Further, the modifications do not affect the stiffness elements uniformly. 


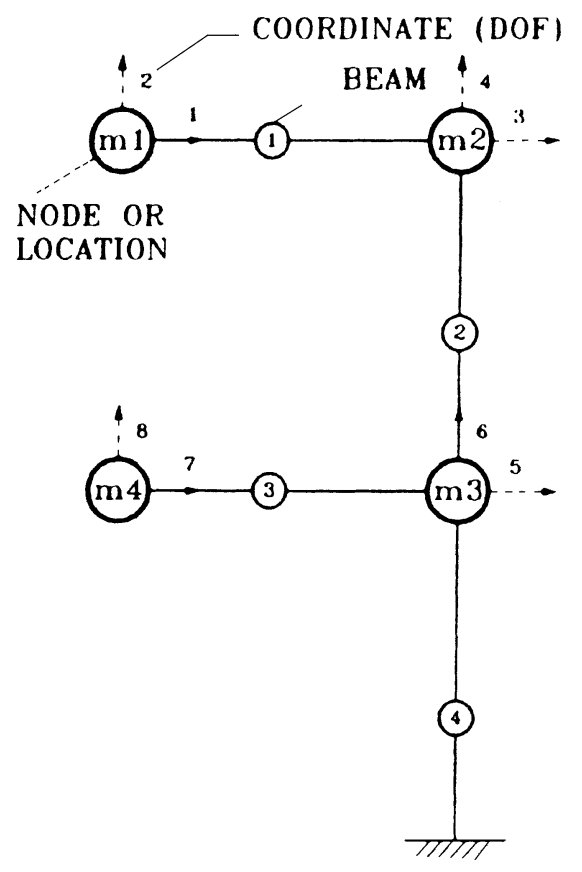

Figure 2. Lumped mass representation of F-type structure.

Accordingly, it is nearly impossible to interpret structural element modifications in terms of a modified stiffness matrix in an exact and simple manner.

In view of the above difficulties, the computation of the stiffness matrix of the modified system is proposed by computing the stiffness modification ratios (or coefficients). This procedure utilizes the dynamicist's knowledge of the analytical model of the dynamic system to be modified. Let the reduced stiffness matrix of this analytical model [AM] be named $\left[K_{\mathrm{AM}}\right]$. Employing the same numerical procedure which has been used to obtain $\left[K_{\mathrm{AM}}\right]$, the stiffness matrix of the modified model [MM], due to structural modification, is obtained. Let this reduced stiffness matrix of the resultant modified model be called $\left[K_{\mathrm{MM}}\right]$. The proposed stiffness modification coefficient matrix $[k]$ can then be obtained as

$$
[k]=\frac{\left[K_{\mathrm{MM}}\right]}{\left[K_{\mathrm{AM}}\right]} .
$$

It may be noted that the right hand side of (14) is not a matrix division, it is simple division of corresponding matrix elements.

Several such modification coefficient matrices can be computed for the standard stiffner/ rib modifications and may be stored in a computer and called on demand, as and when required. The stiffness matrix of the modified system $\left[K_{M}\right]$ can be obtained by using the identified stiffness matrix of the system $[K]$ and coefficient matrix $[k]$ as

$$
\left[K_{\mathrm{M}}\right]=[k] *[K] .
$$

Again $*$ on the right hand side of (15) does not imply a matrix multiplication, it is simple multiplication of corresponding matrix elements.

It may be observed in (14) that as $[k]$ is obtained by finding the ratios, the errors due to the dynamicist's analytical modelling skill which would appear both in the numerator as 
(a)

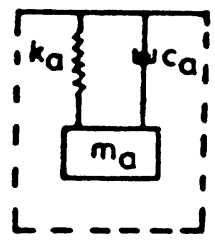

(b)

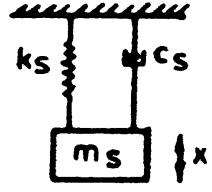

(c)

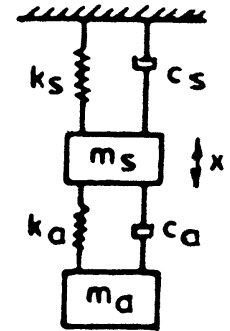

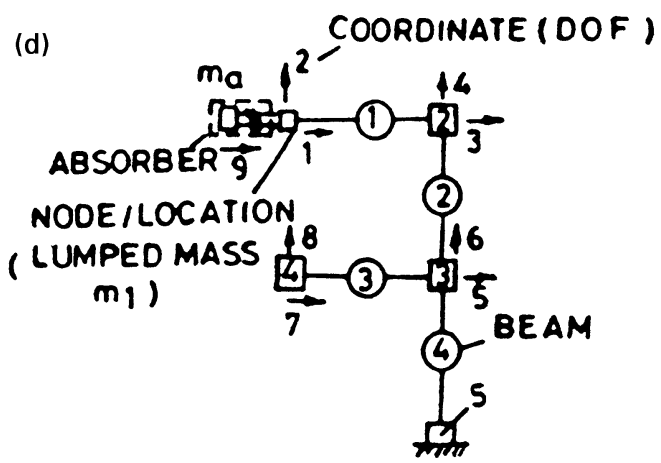

(e)

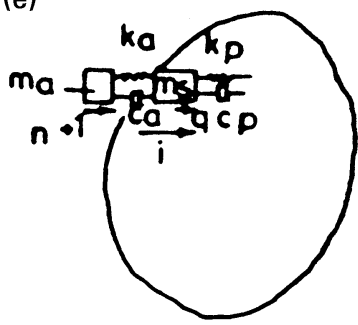

Figure 3. Modifications of systems with absorbers. (a) SDOF absorber, (b) SDOF system, (c) absorber attached to SDOF system, (d) SDOF absorber attached to 8-DOF Fstructure, (e) absorber attached to an $n$-DOF system along coordinate $i$.

well as denominator of the RHS of (14) would tend to cancel out. As a result, coefficients in $[k]$ are expected to be reasonably accurate. The modified structural damping matrix $\left[H_{\mathrm{M}}\right]$ is obtained from $\left[K_{\mathrm{M}}\right]$. The stiffness modifier may have a structural damping coefficient $g_{s}$ which may not be the same as that of the structural element being modified. In any case the following expressions may be used to compute $\left[H_{\mathrm{M}}\right]$ :

$$
\left[H_{\mathrm{M}}\right]=[H]+g_{s}\left(\left[K_{\mathrm{M}}\right]-[K]\right) .
$$

\subsection{Tuned absorber modifications}

Kundra \& Nakra (1987) have determined the matrix changes when a structure is modified by a tuned absorber. A simple tuned absorber is like a single degree of freedom dynamic system shown in figure 3 a with parameters viz. mass $m$, stiffness $k$, viscous damping coefficient $c_{a}$ (or structural damping coefficient $g_{a}$ ) and its natural frequency (or tuned frequency) $\omega_{t}$. The parameters for this single degree of freedom system (SDOF) are related by the well-known relationship,

$$
\omega_{t}=\left(k_{a} / m_{a}\right)^{1 / 2},
$$

where $\omega_{t}$ is tuned/natural frequency of the absorber, $m_{a}$ is its mass and $k_{a}$ is its stiffness.

A tuned absorber is attached in such a manner to the structure of the system that its mass $m_{a}$ vibrates along or parallel to the coordinate of unwanted vibrations. Thus the SDOF system shown in figure $3 \mathrm{~b}$ that has unwanted vibrations along the coordinate $\mathrm{x}$, when 
modified with the absorber of figure 3a, will result in the modified system shown in figure $3 \mathrm{c}$. It may be observed that the modified system has two degrees of freedom, i.e. one degree of freedom more than that of the unmodified system. The eight DOF F-type structure under investigation, when modified with a typical absorber placed along the horizontal coordinate, will have nine degrees of freedom as shown in figure $3 \mathrm{~d}$. In general, an $n$-DOF system will be modified to an $(n+1)$-DOF system. Let $n+1$ represent the number of degrees of freedom of mass $m_{a}$ of the attached absorber along a coordinate $i$ of an $n$-DOF system as shown in figure $3 \mathrm{e}$. The absorber is attached to one of the lumped masses $m_{s}$ (having the DOF number $q$ ) of the structure having $n$ degrees of freedom. The DOF named $q$ is along or parallel to coordinate $i$. The equations of motion of the modified system involving mass $m_{a}$ of the absorber, and the lumped mass $m_{s}$ of the unmodified structure for a sinusoidal excitation along coordinate $i$ acting at mass $m_{s}$ are,

$$
\begin{aligned}
m_{s} \ddot{x}_{q}+\left(k_{a}+k_{p}\right) x_{q}+\left(c_{a}+c_{p}\right) \dot{x}_{q}-k_{a} x_{n+1}-c_{a} \dot{x}_{n+1} & =F \sin \omega t, \\
m_{a} \ddot{x}_{n+1}-k_{a} x_{q}-c_{a} \dot{x}_{q}+k_{a} x_{n+1}+c_{a} \dot{x}_{n+1} & =0,
\end{aligned}
$$

where $k_{p}$ and $c_{p}$ are the values of stiffness and damping, effective along coordinate $i$ for the previously unmodified system and $c_{a}$ is the damping of the absorber. The equations of motion involving other lumped masses may be assumed to remain the same as for the lumped masses of the unmodified system. The equation of motion (18) indicates that mass, stiffness, as well as the damping matrices of the system are affected when an absorber is attached to it. The modified matrices are obtained as explained in the following subsections.

3.4a Determination of modified mass matrix: If (18), along with other lumped masses of the modified system now having $n+1$ degrees of freedom, is put in matrix form, then mass $m_{s}$ would be located in the $(n+1) \times(n+1)$ mass matrix as element number $(q, q)$ as in the unmodified matrix.

Column number : $q$
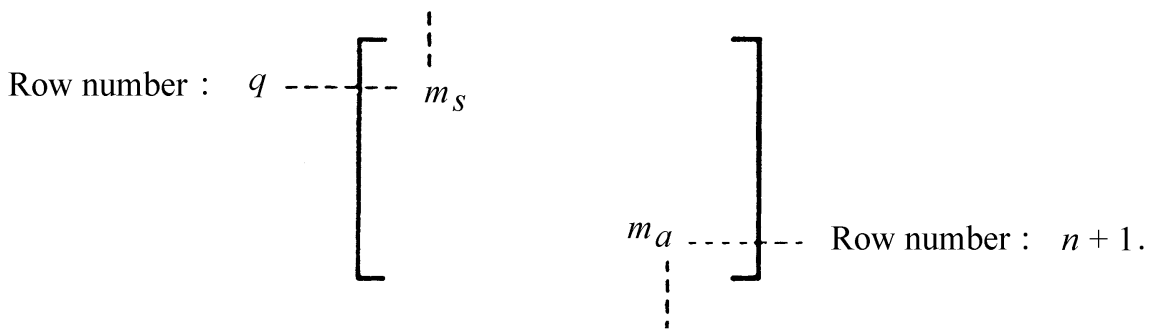

Column number : $n+1$

The absorber's mass would always appear as the $(n+1, n+1)$ th element in the modified matrix. The mass modification matrix is therefore always an $(n+1) \times(n+1)$ matrix with all zero elements except the $(n+1, n+1)$ th element as the mass $m_{a}$ :

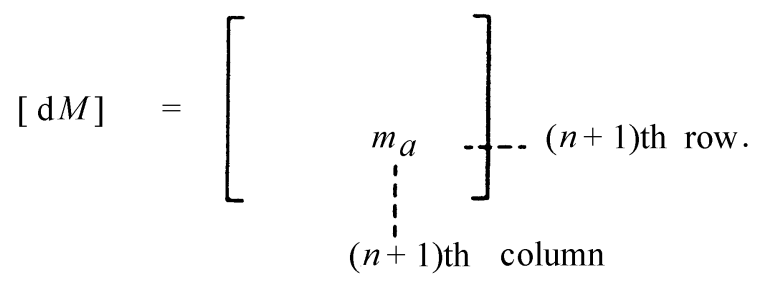


The mass matrix of the modified system is obtained as

$$
\left[M_{M}\right]=\left[M_{+1}\right]+[\mathrm{d} M],
$$

where $\left[M_{+1}\right]$ is obtained by raising the unmodified $n \times n$ element mass matrix [M] to $(n+1) \times(n+1)$ element matrix $\left[M_{+1}\right]$ by inserting zero values along $(n+1)$ th column and row.

$$
\left[M_{+1}\right]=\left[\begin{array}{cc}
{[M]} & 0 \\
0 & 0
\end{array}\right]
$$

3.4b Modified stiffness matrix: Observation of stiffness matrix representation of (18) indicates that the stiffness modification matrix would have entry of $k_{a}$ at location $(q, q)$ and $(n+1, n+1)$ and of $-k_{a}$ at $(n+1, q)$ and $(q, n+1)$.

$$
\begin{gathered}
{[\mathrm{d} K]=\left[\begin{array}{rrr}
\text { Column number : } & q \\
k_{a} & -k_{a} & - \\
-k_{a} & k_{a} & -f-\text { Row number : } q \\
& \vdots
\end{array}\right] \text { - Row number : } n+1 .} \\
\text { Column number : } n+1
\end{gathered}
$$

The modified stiffness matrix is obtained as

$$
\left[K_{M}\right]=\left[K_{+1}\right]+[\mathrm{d} K],
$$

where $\left[K_{+1}\right]$ is obtained by raising the unmodified $n \times n$ element stiffness matrix [K] to $(n+1) \times(n+1)$ element matrix $\left[K_{+1}\right]$ by inserting zero values along $(n+1)$ th column and row.

$$
\left[K_{+1}\right]=\left[\begin{array}{cc}
{[K]} & 0 \\
0 & 0
\end{array}\right]
$$

3.4c Modified structural damping matrix: For the tuned absorber, modification damping matrix may be obtained as

$$
[\mathrm{d} H]=g_{a} *[\mathrm{~d} K],
$$

and the structural damping matrix of the modified system is then determined as

$$
\left[H_{M}\right]=\left[H_{+1}\right]+[\mathrm{d} H],
$$

where $\left[H_{+1}\right]$ is obtained from $[H]$ of the unmodified system as

$$
\left[H_{+1}\right]=\left[\begin{array}{cc}
{[H]} & 0 \\
0 & 0
\end{array}\right] .
$$

To predict the response of the absorber modified system the equations of motion (1)-(5) with modified matrices, viz. $\left[M_{\mathrm{M}}\right],\left[H_{\mathrm{M}}\right]$, and $\left[K_{\mathrm{M}}\right]$ can be employed. 


\section{Efficient reanalysis methods}

These methods help to evaluate quickly the desired effect of a modification instead of complete re-solution of (4)-(6). Rayleigh's method is widely used for eigenvalue reanalysis and is shown below to illustrate the point

$$
\Delta \lambda_{M}=\left(\Omega_{r}+\mathrm{d} \Omega_{r}\right)^{2}=\frac{\left\{\phi_{r}\right\}^{T}[K+\mathrm{d} K]\left\{\phi_{r}\right\}}{\left\{\phi_{r}\right\}^{T}[M+\mathrm{d} M]\left\{\phi_{r}\right\}} .
$$

For small modifications (26) can be simplified as

$$
\mathrm{d} \Omega_{r}=\Omega_{r}\left\{\frac{\left\{\phi_{r}\right\}^{T}[\mathrm{~d} K]\left\{\phi_{r}\right\}}{2\left\{\phi_{r}\right\}^{T}[K]\left\{\phi_{r}\right\}}-\frac{\left\{\phi_{r}\right\}^{T}[\mathrm{~d} M]\left\{\phi_{r}\right\}}{2\left\{\phi_{r}\right\}^{T}[M]\left\{\phi_{r}\right\}}\right\} .
$$

It can be observed from (27) that for any changes of $[\mathrm{d} K]$ and $[\mathrm{d} M]$, changes in $\Omega$ can be approximately but readily calculated. For only mass or only stiffness modification the equation could also be used for synthesis, that is for determining $[\mathrm{d} M]$ or $[\mathrm{d} K]$ for desired small changes of natural frequency. It is obvious that it is the assumption of small changes in $\Omega$ and the assumption about the nature of modifications, i.e. $[\mathrm{d} K]$ or $[\mathrm{d} M]$ in this case, which makes reanalysis and synthesis quicker and more efficient as compared to the complete solution of (5). Even the transformation of (5) to modal coordinates does not offer any advantage for reanalysis (Baldwin \& Hutton 1985). Several SDM formulations based on a variety of simplifications have emerged. One such simplification reported (Shen \& Stevens 1989; Ravi et al 1994) is given as

$$
\begin{aligned}
& \Delta \lambda_{i} \approx \frac{\{\phi\}_{i}^{T}\left[\Delta K-\lambda_{i} \Delta M\right]\{\phi\}_{i}}{\{\phi\}_{i}^{T}[M]\{\phi\}_{i}}, \\
& \{\Delta \phi\}_{i} \approx \sum_{\substack{j=1 \\
i \neq j}}^{n} P_{i j}\{\phi\}_{j},
\end{aligned}
$$

where

$$
p_{i j}=\frac{\{\phi\}_{j}^{T}\left[\Delta K-\lambda_{i} \Delta M\right]\{\phi\}_{i}}{\{\phi\}_{i}^{T}\left(\lambda_{i}-\lambda_{j}\right)\{\phi\}_{i}^{T}[M]\{\phi\}_{i}} .
$$

SDM methods based on above perturbation equations for modification of beams and beam-based structures with free-layer and constrained-layer viscoelastic materials have been developed (Ravi et al 1994, 1995).

\section{SDM directly from frequency response functions}

These methods start from a set of experimentally obtained frequency response functions (FRFs) and do not need identification of either a modal or a spatial model of the structure (Sestieri \& D'Ambrogio 1989). The FRF could be receptance, mobility or inertance. The dynamic stiffness matrix (DSM) is determined from the inverse of the FRF matrix,

$$
[s(\omega)]=[H(\omega)]^{-1} .
$$


The DSM of the modified structure can be obtained by formulating a modification matrix $[\Delta s(\omega)]$ and adding the same to $[s(\omega)]$. The response FRF of the modified structure $\left[H_{m}(\omega)\right]$ may then be predicted as

$$
\left[H_{m}(\omega)\right]=[[s(\omega)]+[\Delta s(\omega)]]^{-1} .
$$

Several matrices $[\Delta s(\omega)]$ for the modifications between coordinates of interest have been developed by researchers. Lumped mass, axial spring, viscous damper, dynamic absorber and continuous rod modification matrices have been reported (Sestieri \& D'Ambrogio 1989). $[\Delta s(\omega)]$ for a transverse beam (Sharma 1993) and a damped beam (Srivastava \& Kundra 1993) have also been developed.

\section{SDM and optimization}

By employing sensitivity derivatives based on optimal modification for desired eigenvalues or other parameters, SDM computer time is reduced. Fox \& Kapoor (1968) have given the derivatives of eivenvalues, which can be derived (Srivastava 1990) in terms of modal parameters and structure's matrices (modified or otherwise), with respect to a design parameter $p_{k}$ as,

$$
\frac{\delta \lambda_{r}}{\delta p_{k}}=\left\{\phi_{r}\right\}^{T}\left[\frac{\delta[K]}{\delta p_{k}}-\lambda_{r} \frac{\delta[M]}{\delta p_{k}}\right]\left\{\phi_{r}\right\} .
$$

The sensitivity of the natural frequency is accordingly written as

$$
\frac{\delta \Omega_{r}}{\delta p_{k}}=\frac{1}{2 \Omega_{r}}\left\{\phi_{r}\right\}^{T}\left[\frac{\delta[K]}{\delta p_{k}}-\Omega_{r}^{2} \frac{\delta[M]}{\delta p_{k}}\right]\left\{\phi_{r}\right\} .
$$

Several modification algorithms for finding optimal values of discrete modification elements namely mass, stiffness and beam elements have been developed (Srivastava \& Kundra 1995) for shifting natural frequency of a structure within specified constraints. Optimal modification employing constrained damping layer treated beams to identify most effective thicknesses of viscoelastic layers for desired natural frequency have also been proposed (Ravi 1994).

A single-step perturbation method, reported separately in detail, has been developed which makes SDM very fast. The method is based upon the following eigenparameter perturbations,

$$
\Delta \lambda_{i}=\frac{\{\psi\}_{i}^{T}\left[\Delta K-\lambda_{i} \Delta M\right]\{\psi\}_{i}}{\{\psi\}_{i}^{T}[M+\Delta M]\{\psi\}_{i}},
$$

where $\{\psi\}$ is the unit-normalised eigenvector.

For eigenvector perturbation, the above updated eigenvalues are employed. The change in the $i$ th eigenvector is given by

$$
\{\Delta \psi\}_{i}=\sum_{\substack{i=1 \\ j \neq i}}^{n} c_{i j}\{\psi\}_{j},
$$


where

$$
c_{i j}=\frac{\{\psi\}_{j}^{T}\left[\Delta K-\lambda_{i}^{*} \Delta M\right]\{\psi\}_{i}}{\left(\lambda_{i}^{*}-\lambda_{j}\right)\{\psi\}_{i}^{T}[M]\{\psi\}_{i}} .
$$

In this faster method, the entire modification of the structure is considered in a single step.

\section{Some SDM results}

The structure considered for these results has been shown in figure 1. The test data (FRF) required for the structure have been generated through computer simulation by modelling this structure as shown in figure 2 for obtaining the matrices $[M]$ and $[K]$, with limited eight coordinates of interest. The structural damping matrix is obtained from $[K]$ by assuming the structural damping coefficient. In some studies, errors are introduced into the simulations to make them closer to actual tests (Kundra \& Nakra 1994).

Effects of SDM with several types of modifiers on dynamic characteristics of the F-type of structure have been studied. Illustrative results of mass, material, beam and tuned absorber modifications with the help of algorithms developed in $\S 3$ on a typical F-type structure consisting of four beams each of $0.3 \times 0.025 \times 0.025 \mathrm{~m}$ are presented. The first natural frequency of the unmodified $\mathrm{F}$ structure is predicted to be $172 \mathrm{rad} / \mathrm{s}$.

The effect of adding to and removing a mass of $2 \mathrm{~kg}$ from the unmodified $\mathrm{F}$ structure at location 1, on the dynamic charcteristics, namely response and natural frequency, is shown in figure 4 . Addition of mass lowers the natural frequency as expected. The response

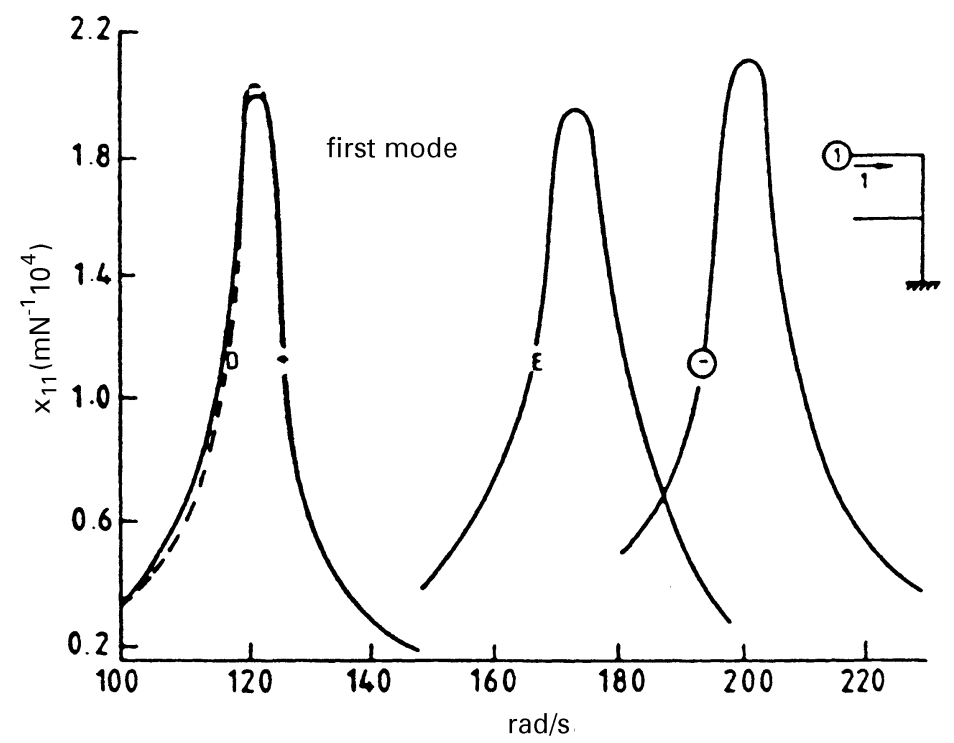

Figure 4. Prediction of dynamic characteristics of F-type structure with mass modifications. - - $\square--2 \mathrm{~kg}$ at location 1 (exact model); - - $-2 \mathrm{~kg}$ at location 1 (DTmodel); - $\ominus-0.5 \mathrm{~kg}$ at location 1 (DT-model); - E - exact model without modification. 


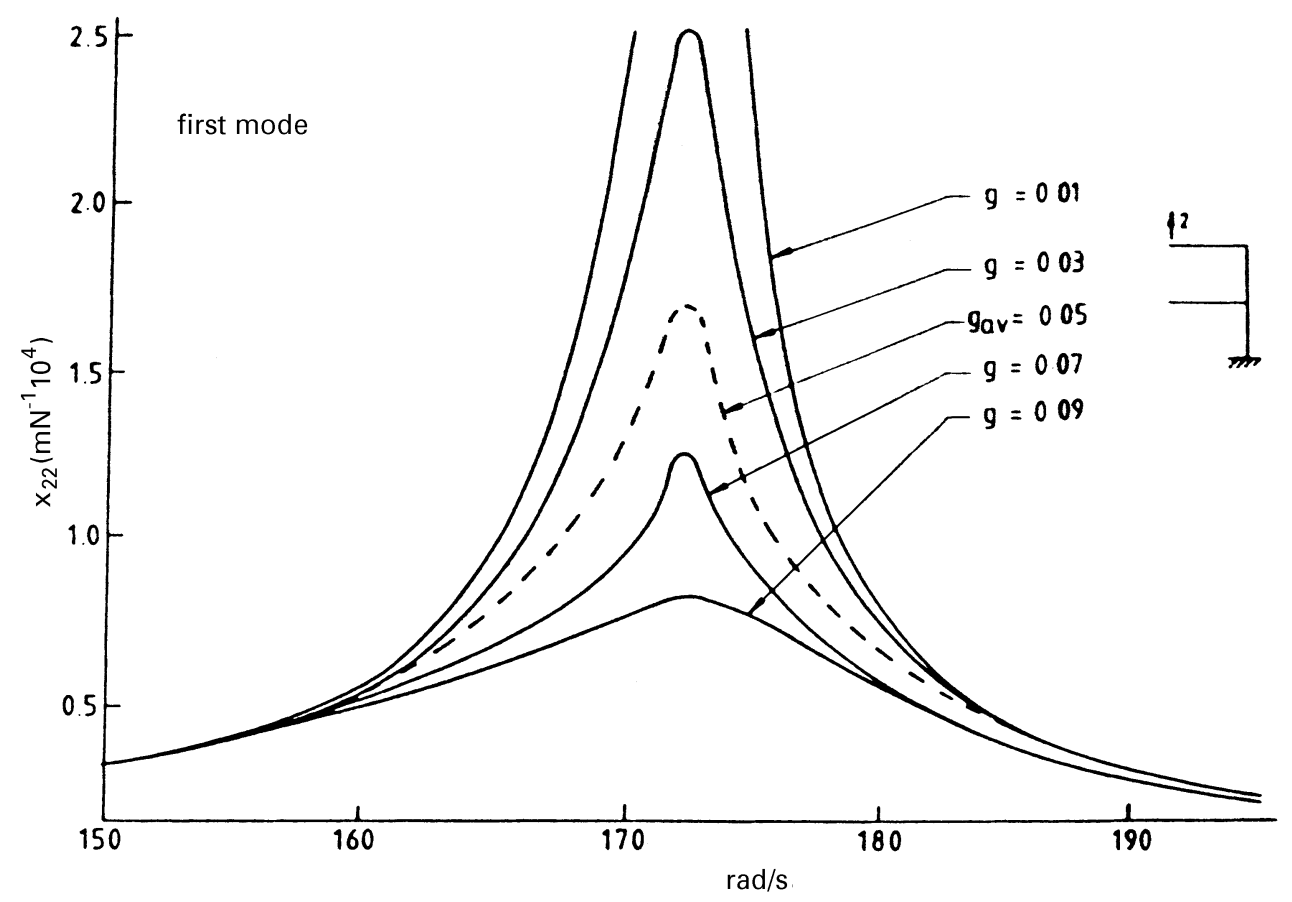

Figure 5. Influence of overall structural damping modifications.

level in this modification gets raised. The figure also shows the prediction obtained with the DT (Dynamic Test) model on introducing errors into the computer simulated test data. The predictions on modifying the material of the structure by materials having different structural damping coefficients are shown in figure 5 which are again on expected lines.

A comparison of the effect of stiffening each of the beams of the F-type structure is shown in figure 6. From the response point of view, stiffening of beam 4 appears to be the most promising provided the shifting of natural frequency to $260 \mathrm{rad} / \mathrm{s}$ is acceptable. The stiffening of beam 2 reduces vibration level while retaining the natural frequency closely. Stiffening of beams 1 or 3 is accompanied by the influence of the mass of stiffeners.

A typical result of modification study on the F-type structure with a tuned absorber is shown in figure 7 . The tuned absorber with a mass of $0.366 \mathrm{~kg}$ and a natural frequency of $172 \mathrm{rad} / \mathrm{s}$ is chosen to match with the first natural frequency of the structure. As anticipated the SDM algorithm predicts that this modification would lead to a lower vibration level but two resonance peaks viz. at $145 \mathrm{rad} / \mathrm{s}$ and $203 \mathrm{rad} / \mathrm{s}$. The effect of attaching the absorber along several coordinates of interest on the response levels, natural frequency and structural stability, has also been studied.

\section{Concluding remarks}

This discussion indicates that structural dynamic modification via models is a potential strategy for achieving the desired dynamic design of structures. Model adjustments of analytical models where system identification is unable to extract a mathematical model 


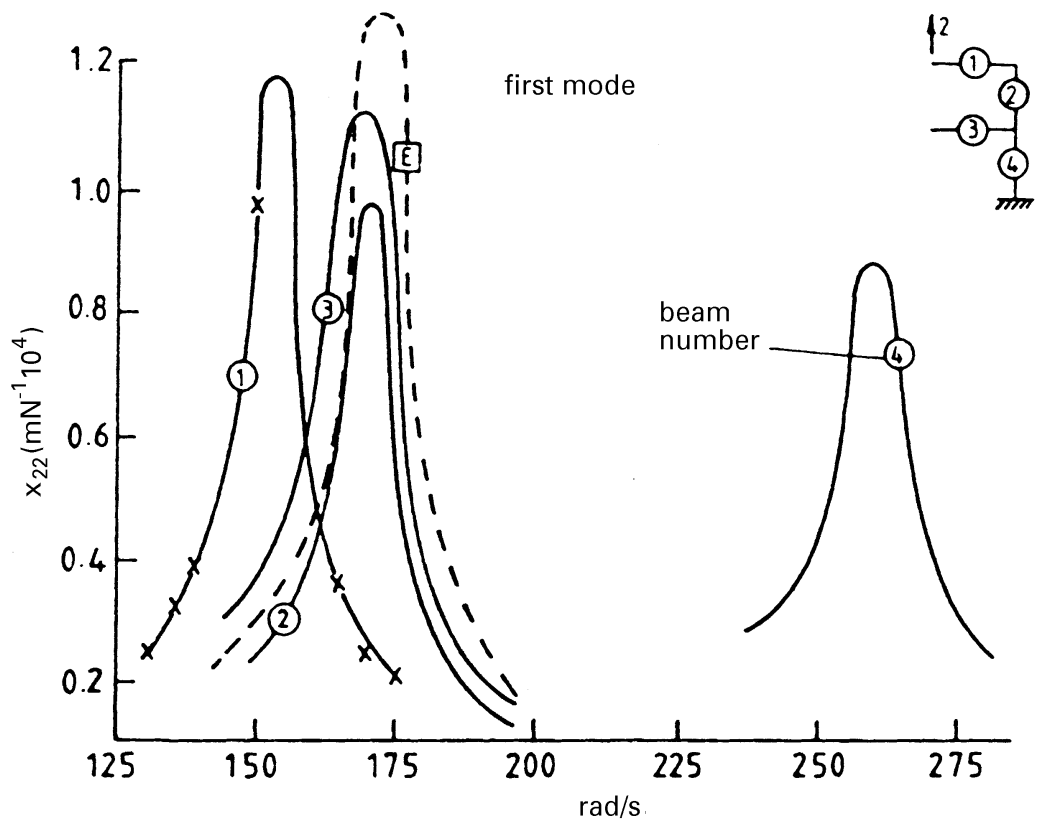

Figure 6. Comparison of influence of stiffening each beam of the F-type structure. - -E- - exact response of F-structure without any modification; $\times \times \times$ response of exact model when beam no. 1 is stiffened to raise its inertia by three; (b) = (2), (3), (4) response of modified F-structure when beam (b) is stiffened to raise its inertia by three (each unmodified beam has same MOI).

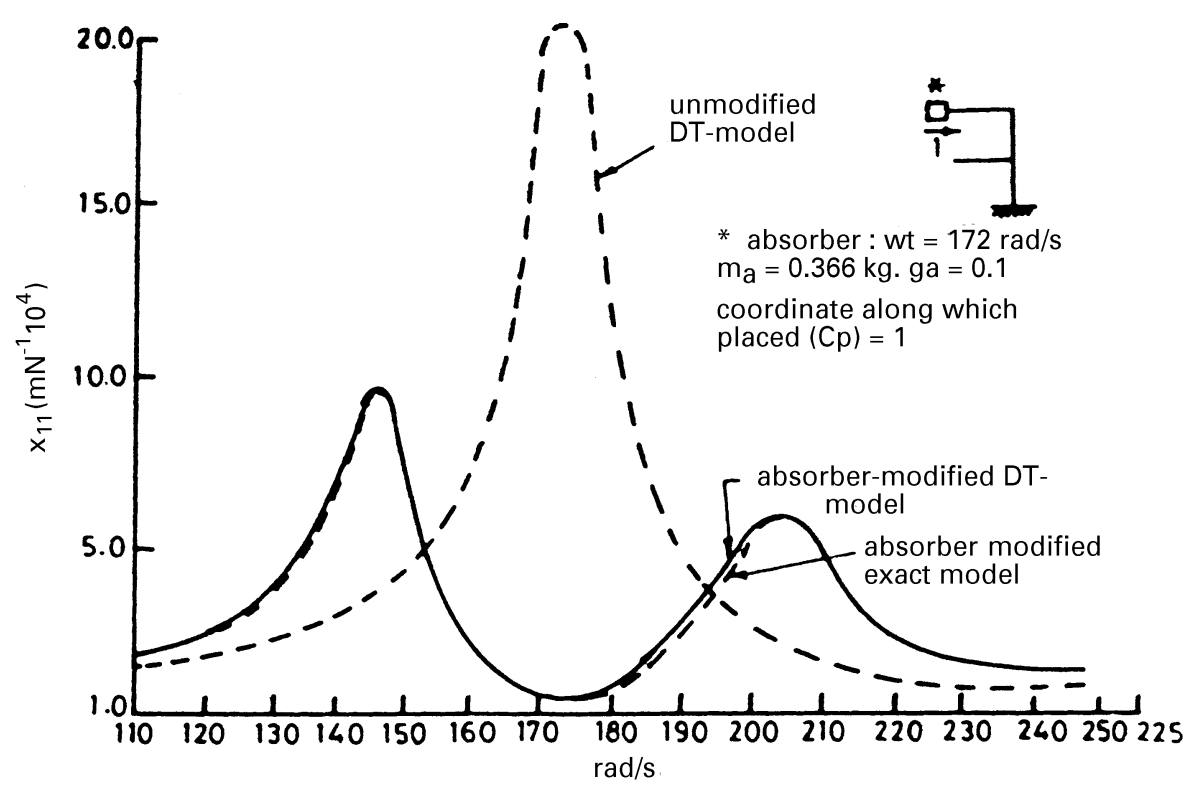

Figure 7. Modification of the F structure with a tuned absorber. 
provides the necessary foundation to make the implementation of these techniques feasible. The principles of perturbation enhance the efficiency of computational effort involved and in addition, the question of SDM based design can be addressed. Further, several optimization techniques including sensitivity analysis are integrable with SDM to meet the objective of optimal dynamic design.

The relationship between several real life modifiers like plates, complex vibration dampers and stiffeners of different shapes and the changes in parametric matrices of complex structures have to be developed. The updated model based SDM also needs to be investigated for covering all the dynamic characteristics of the structures. The multiparameter optimization techniques should be extended to SDM to obtain simultaneously the optimal values of all the design variables constituting possible modifications. This should finally lead to the development of optimal dynamic design algorithms based on SDM.

\section{References}

Baldwin J F, Hutton S G 1985 Natural modes of modified structures. AIAA J. 23: 1737-1743

Berman A 1975 Determining structural parameters from dynamic testing. Shock Vibr. Dig. 7: 10-17 Brandon J A 1990 Strategies for structural dynamic modification (New York: John Wiley)

Craig R R Jr 1985 A review of time-domain and frequency-domain component mode synthesis methods. ASME: Combined Exp./Anal. Model. Dyn. Syst. AMD-67: 1-30

Ewins D J 1984 Modal testing: Theory and practice (Somerset: Research Studies Press)

Fox R L, Kapoor M P 1968 Rates of change of eigenvalues and eigenvectors. AIAA J. 6: 2426-2429

Imergun M, Visser W J 1991 A review of model updating techniques. Shock Vibr. Dig. 23: 9-20

Kundra T K 1986 Studies in identification and modification of dynamic mechanical systems. $\mathrm{PhD}$ thesis, Indian Institute of Technology, Delhi

Kundra T K, Nakra B C 1987 Structural modification with a tuned absorber attached to a complex structure via identified models. ASME: Modal Testing Anal. DE-3: 37-42

Kundra T K, Nakra B C 1994 Influence of dynamic test errors on system identification and modification. J. Inst. Eng. (India) 75: 14-18

Luk Y W, Mitchell L D 1984 Implementation of the dual space structural modification method. Proc. 2nd Int. Modal Analysis Conference (Orlando) 2: 930-936

Natke H G (ed.) 1982 Identification of vibrating structures (New York: Springer Verlag, Wein)

Rao S S 1989 Optimum design of structures under shock and vibration environment. Shock Vibr. Dig. 21(7):

Ravi S S A 1994 Structural dynamic modifications and design sensitivity analysis of structures with viscoelastic damping treatment. Ph D thesis, Indian Institute of Technology, Delhi

Ravi S S A, Kundra T K, Nakra B C 1994 Eigenvalue reanalysis of sandwich beams with viscoelastic core using perturbation method. Modal Anal.: Int. J. Anal. Exp. Modal Anal. 9: 203-217

Ravi S S A, Kundra T K, Nakra B C 1995a A response reanalysis of damped beams using eigenparameter perturbation. J. Sound Vibr. 179: 399-412

Ravi S S A, Kundra T K, Nakra B C 1995b A response reanalysis of damped complex beam structures. Proc. Int. Modal Analysis Conference (IMAC-XII) (Tennessee)

Sestieri A, D'Ambrogio W 1989 A modification method for vibration control of structures. Mech. Syst. Signal Process. 3: 229-253

Sharma K 1993 Dynamic improvement by structural dynamic modifications. M Tech thesis, Indian Institute of Technology, Delhi

Shen S, Stevens K K 1989 A perturbation method for the analysis of structural systems. Proc. of DAMPING-89 (Florida) pp 1-16

Snyder V W 1985 Structural modification and modal analysis. Exp. Tech. 9: 245-315 
Srivastava R K 1990 Modal identification and optimal modification of systems via dynamic stiffness and sensitivity analysis. Ph D thesis, Indian Institute of Technology, Delhi

Srivastava R K, Kundra T K 1993 Structural dynamic modification with damped beam elements. Comput. Struct. 48: 943-950

Srivastava R K, Kundra T K 1995 Optimal modification of dynamic systems with a natural frequency constraint. Proc. Int. Modal Analysis Conference (IMAC-XII) (Tennessee) 